\title{
Carbon-13 Labeling for Improved Tracer Depth Profiling of Organic Materials Using Secondary Ion Mass Spectrometry
}

\author{
S. E. Harton \\ Department of Materials Science and Engineering, North Carolina State University, Raleigh, North Carolina, \\ USA
}

\author{
F. A. Stevie \\ Analytical Instrumentation Facility, North Carolina State University, Raleigh, North Carolina, USA
}

H. Ade

Department of Physics, North Carolina State University, Raleigh, North Carolina, USA

\begin{abstract}
${ }^{13} \mathrm{C}$ labeling is introduced as an alternative to deuterium labeling for analysis of organic materials using secondary ion mass spectrometry (SIMS). A model macromolecular system composed of polystyrene (PS) and poly(methyl methacrylate) (PMMA) was used to compare the effects of isotopic labeling using both deuterium substitution (dPS) and ${ }^{13} \mathrm{C}$ labeling $\left({ }^{13} \mathrm{C}\right.$-PS). Clear evidence is shown that deuterium labeling does introduce changes in the thermodynamic properties of the system, with the observation of segregation of dPS to an hPS:dPS/hPMMA interface. This type of behavior could significantly impact many types of investigations due to the potential for improper interpretation of experimental results as a consequence of labeling-induced artifacts. ${ }^{13} \mathrm{C}$ labeling is shown to provide a true tracer for analysis using SIMS. (J Am Soc Mass Spectrom 2006, 17, 1142-1145) (C) 2006 American Society for Mass Spectrometry
\end{abstract}

S econdary ion mass spectrometry (SIMS) has become a powerful analysis tool for depth profiling or chemical mapping of organic and biological systems [1-5]. One such application has been onedimensional depth profiling of polymer films and multilayers, which has provided a significant driving force for growth in experimental and theoretical polymer physics over the past 20 years [6]. Deuterium substitution is by far the most common type of tracer labeling used for analysis of polymer films and multilayers [7], with the commercial availability of various labeled reagents and monomers. Unfortunately, it can introduce changes in the properties being measured. For instance, it has been established that deuterium substitution can affect polymer/polymer phase behavior, with reported changes in both bulk [8-10] and surface interactions [11, 12], and deuterium substitution has even been shown to alter polymer-solvent phase behavior [13]. More recently, it has been found that deuterium substitution can have a profound effect on the proper-

Published online May 30, 2006

Address reprint requests to Professor H. Ade, Department of Physics, North Carolina State University, Raleigh, NC 27695, USA. E-mail: harald_ade@ ncsu.edu ties of a polymer/polymer heterogeneous interface, with the observation of diffusion-controlled segregation of deuterium labeled polystyrene (dPS) to an hPS:dPS / poly(methyl methacrylate) (hPMMA) interface [14]. This type of behavior could significantly impact several types of investigations, such as drug delivery mechanisms [15], with the potential for improper interpretation of experimental results due to labeling-induced artifacts. Alternative methods of tracer labeling are therefore required. Here, we demonstrate the development of one such method using ${ }^{13} \mathrm{C}$ labeling, with the expectation that this method will be generally applicable for analysis of various carbonaceous systems in fields ranging from polymer physics to biotechnology.

\section{Experimental}

\section{Materials and Sample Preparation}

For the investigation of dPS segregation to an hPS:dPS/ hPMMA interface, the polymers were purchased from Polymer Source (Dorval, Canada), and are listed in Table 1 along with the glass transition temperatures $\left(T_{\mathrm{g}}\right)$ as determined using differential scanning calorimetry. hPMMA was cast from 1-chlorobenzene onto hydrogen- 
Table 1. Polymer properties

\begin{tabular}{lccr}
\hline Polymer & $\mathrm{M}_{\mathrm{w}} / \mathrm{kDa}$ & $\mathrm{M}_{\mathrm{w}} / \mathrm{M}_{\mathrm{n}}$ & $\mathrm{T}_{\mathrm{g}} /{ }^{\circ} \mathrm{C}$ \\
\hline \hline $\mathrm{dPS}$ & 82.8 & 1.15 & 97 \\
$\mathrm{hPS}$ & 73.0 & 1.04 & 96 \\
${ }^{13} \mathrm{C}-\mathrm{PS}$ & 79.4 & 1.20 & 100 \\
${ }^{12} \mathrm{C}-\mathrm{PS}$ & 73.7 & 1.19 & 100 \\
$\mathrm{hPMMA}$ & 155 & 1.05 & 127 \\
${ }^{12} \mathrm{C}-\mathrm{PMMA}$ & 90.9 & 1.26 & 100 \\
\hline
\end{tabular}

passivated Si (100) to a thickness of ca. $200 \mathrm{~nm}$ and annealed at $150{ }^{\circ} \mathrm{C}$ for $3 \mathrm{~h}$. PS $(90 \% \mathrm{hPS}+10 \%$ dPS $\mathrm{vol} / \mathrm{vol}$ ) was cast directly onto hPMMA from 1-chloropentane, a selective solvent for PS over PMMA, to a thickness of $210 \mathrm{~nm}$ and annealed for $94 \mathrm{~h}$ at $138^{\circ} \mathrm{C}$, which is a much longer time than required for equilibrium segregation of this system [14]. A $50 \mathrm{~nm}$ (approximate) hPS sacrificial layer was added to the sample before analysis to avoid pre-equilibrium sputtering at the hPS: dPS surface. For the novel ${ }^{13} \mathrm{C}$ depth profiling investigation, ${ }^{13} \mathrm{C}$-PS, ${ }^{12} \mathrm{C}$-PS, and ${ }^{12} \mathrm{C}-\mathrm{PMMA}$ were synthesized using atom transfer radical polymerization (ATRP) with previously outlined conditions [16], and are listed in Table 1. A mixture of $33 \% \alpha, \beta-{ }^{13} \mathrm{C}$ labeled styrene (Isotec) and unlabeled styrene monomer were used in the synthesis of ${ }^{13} \mathrm{C}$-PS resulting in $10.3 \%{ }^{13} \mathrm{C}$ relative to the total carbon in the ${ }^{13} \mathrm{C}-\mathrm{PS}$, as determined using time-of-flight SIMS (PHI TRIFT I). ${ }^{12} \mathrm{C}$-PS and ${ }^{12} \mathrm{C}$-PMMA have $1.1 \%{ }^{13} \mathrm{C}$ (natural abundance). For the first set of samples, ${ }^{13} \mathrm{C}-\mathrm{PS}$ was cast onto $\mathrm{Si}(100)$ that had a $2 \mathrm{~nm}$ native oxide $\left(\mathrm{SiO}_{x}\right)$ layer to a thickness of $180 \mathrm{~nm}$ and annealed at $125^{\circ} \mathrm{C}$ for $24 \mathrm{~h}$. A second set of samples was prepared to evaluate the effects of isotopic labeling at the PS/PMMA interface. ${ }^{12} \mathrm{C}-$ PMMA was cast onto $\mathrm{Si}(100)$ that had a $2 \mathrm{~nm} \mathrm{SiOx} \mathrm{layer}$ and was annealed at $125{ }^{\circ} \mathrm{C}$ for $30 \mathrm{~min}$. Next, $5 \%{ }^{13} \mathrm{C}$-PS + $95 \%{ }^{12} \mathrm{C}-\mathrm{PS}$ (vol/vol, solvent free basis) was cast onto the PMMA from 1-chloropentane to a thickness of $175 \mathrm{~nm}$ and annealed at $125{ }^{\circ} \mathrm{C}$ for $24 \mathrm{~h}$. A $125 \mathrm{~nm}$ layer of ${ }^{12} \mathrm{C}$-PS was floated onto both of these sets of samples to establish the background (natural abundance) ${ }^{13} \mathrm{C}$ secondary ion yields.

\section{Secondary Ion Mass Spectrometry}

All depth profiles were performed using a CAMECA IMS-6f magnetic sector mass spectrometer (Courbevoie, France), which has the mass resolving capabilities to completely separate ${ }^{12} \mathrm{C}^{1} \mathrm{H}(13.00,782 \mathrm{Da})$ from ${ }^{13} \mathrm{C}$ $(13.00,335 \mathrm{Da})[4,17]$. A $20 \mathrm{~nm}$ Au coating was sputtered onto the samples before SIMS analysis to help minimize charging. Analysis conditions for $\mathrm{O}_{2}^{+}$primary ion bombardment included a $30 \mathrm{nA}$ primary current rastered over a $180 \mu \mathrm{m} \times 180 \mu \mathrm{m}$ area, with $5.5 \mathrm{keV}$ impact energy ( $10 \mathrm{kV}$ primary with $4.5 \mathrm{kV}$ sample bias) and a mass resolution $(\mathrm{m} / \Delta \mathrm{m})$ of 3000 . The angle of incidence for the primary ions was $41^{\circ}$ from normal. Positive secondary ions were detected from a $60 \mu \mathrm{m}$ diameter optically gated area positioned in the center of the raster. For $\mathrm{Cs}^{+}$, analysis conditions included a $10 \mathrm{nA}$ primary current rastered over a $180 \mu \mathrm{m} \times 180 \mu \mathrm{m}$ area, with $6.0 \mathrm{keV}$ impact energy $(5 \mathrm{kV}$ primary with $-1 \mathrm{kV}$ sample bias) and $\mathrm{m} / \Delta \mathrm{m}=2910$. The angle of incidence for the primary ions was $27^{\circ}$ from normal. Negative secondary ions were detected from a $60 \mu \mathrm{m}$ diameter optically gated area positioned in the center of the raster.

\section{Results and Discussion}

Figure 1 shows an example of the effect of deuterium labeling at the heterogeneous hPS:dPS/hPMMA polymer/polymer interface. Because dPS has an energetic preference for hPMMA, there is an observed segregation of dPS to the hPS:dPS/hPMMA interface [14]. The $\mathrm{hPS}$ :dPS blend is well within the single-phase region of the hPS:dPS phase diagram [10], so the observed phenomenon is not due to bulk phase separation. This behavior has been described in detail elsewhere, with identification of diffusion-controlled behavior at very early times [14]. Figure 2 shows profiles of the ${ }^{12} \mathrm{C}$-PS/ ${ }^{13} \mathrm{C}-\mathrm{PS}$ bilayer using both $6.0 \mathrm{keV} \mathrm{Cs}^{+}$(Figure 2a and c) and $5.5 \mathrm{keV} \mathrm{O}_{2}^{+}$(Figure $2 \mathrm{~b}$ and $\mathrm{d}$ ) primary ion bombardment. In both profiles it is evident that the ${ }^{13} \mathrm{C}$ is indeed completely mass resolved, as the ${ }^{12} \mathrm{C}^{1} \mathrm{H}$ profile clearly traces the ${ }^{12} \mathrm{C}$ profile and not the ${ }^{13} \mathrm{C}$ profile. Figure $2 \mathrm{c}$ and $\mathrm{d}$ show the profiles for the volume fraction of ${ }^{13}$ C-PS $(\varphi)$ as a function of effective depth $(z)$, based on the PS sputtering rates, as determined by:

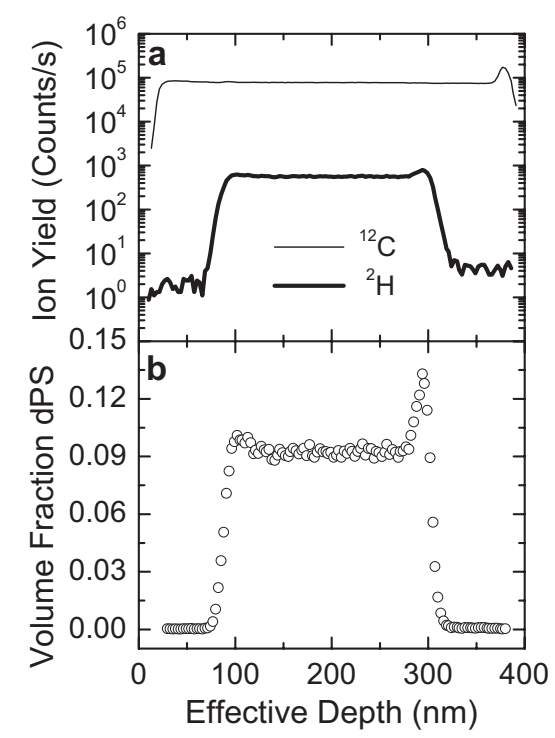

Figure 1. Segregation of dPS to the hPS:dPS/hPMMA interface and hPS:dPS surface after annealing for $94 \mathrm{~h}$ at $138^{\circ} \mathrm{C}$. Both the relative count rates (a) and dPS volume fraction (b) are presented. The segregation of dPS to the hPS:dPS/hPMMA interface clearly shows that dPS has an energetic preference for hPMMA. The hPS:dPS blend is well within the single phase region of the phase diagram. SIMS analysis was performed using $5.5 \mathrm{keV}$ impact energy $\mathrm{O}_{2}^{+}$ion bombardment with detection of positive secondary ions. The effective depth was determined by assuming a constant PS sputtering rate throughout the analyzed film assembly. 

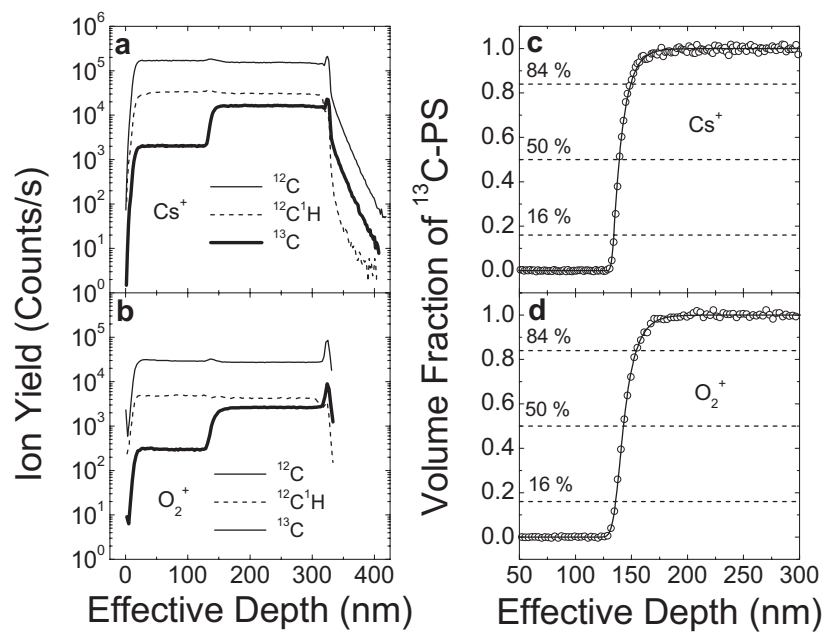

Figure 2. SIMS depth profiles showing ${ }^{12} \mathrm{C}$ (solid line), ${ }^{12} \mathrm{C}^{1} \mathrm{H}$ (dashed line), and ${ }^{13} \mathrm{C}$ (bold line) secondary ion yields and (c, d) volume fraction of ${ }^{13} \mathrm{C}$-PS in the ${ }^{12} \mathrm{C}$-PS $/{ }^{13} \mathrm{C}$-PS bilayer. Both $(\mathbf{a}, \mathbf{c})$ $6.0 \mathrm{keV} \mathrm{Cs}{ }^{+}$and $(\mathbf{b}, \mathbf{c}) 5.5 \mathrm{keV} \mathrm{O}_{2}^{+}$primary ion bombardment, with detection of negative and positive secondary ions, respectively, were used for analysis of this sample. Shown in (c) and (d) are the midpoint (50\%) and 16 and $84 \%$ intensity lines, which are used to semiquantitatively describe the asymmetry of the profiles (see Table 2). The solid lines in (c) and (d) are a fit using a step function convoluted with a Gaussian and an exponential function (see eqs 2-4). The effective depth was determined by assuming a constant PS sputtering rate throughout the film assembly.

$$
\phi(z)=\frac{Y_{13}(z)-Y_{N A}}{Y_{P}-Y_{N A}}
$$

where $Y_{13}(z)$ is the ${ }^{13} \mathrm{C}$ ion yield, normalized to the total $\mathrm{C}\left({ }^{12} \mathrm{C}+{ }^{13} \mathrm{C}\right)$ ion yield, at depth $z, Y_{N A}$ is the normalized ${ }^{13} \mathrm{C}$ ion yield of pure ${ }^{12} \mathrm{C}$-PS (natural abundance), and $Y_{P}$ is the normalized ${ }^{13} \mathrm{C}$ ion yield of pure ${ }^{13} \mathrm{C}$-PS. Under the conditions implemented here for both $\mathrm{O}_{2}^{+}$ and $\mathrm{Cs}^{+}$bombardment, excellent signal-to-noise (S/N) is observed in the profiles shown in Figure $2 \mathrm{c}$ and $\mathrm{d}$, even though there is only $10.3 \%$ total ${ }^{13} \mathrm{C}$, based on ${ }^{13} \mathrm{C} /\left({ }^{13} \mathrm{C}+{ }^{12} \mathrm{C}\right)$, in the ${ }^{13} \mathrm{C}$-PS. The profiles shown in

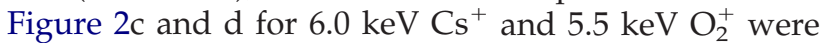
evaluated using an $84 / 16$ analysis $(2 \sigma)_{84 / 16}$, and the standard deviation using 50/16 $\left(\sigma_{50 / 16}\right)$ and $84 / 50$ $\left(\sigma_{84 / 50}\right)$ analyses, as shown in Table 2.

The profile lineshapes and nearly a factor of two difference between $\sigma_{50 / 16}$ and $\sigma_{84 / 50}$ observed through the ${ }^{12} \mathrm{C}$-PS $/{ }^{13} \mathrm{C}$-PS interface clearly demonstrate that these profiles cannot be accurately represented by a symmetric Gaussian instrument resolution convoluted with a sharp intrinsic profile. The observed convolution-type arises primarily from ion-induced mixing of the ${ }^{13} \mathrm{C}$ and ${ }^{12} \mathrm{C}$ matrix and implantation of the ${ }^{13} \mathrm{C}$ further into the film (tailing) [18]. Analogous behavior has been observed for ${ }^{30} \mathrm{Si}$ implants in a ${ }^{28} \mathrm{Si}$ matrix [18]. Here we employ a simplified version of a convolution scheme that has been outlined previously [18, 19]. It combines a Gaussian with a standard deviation $\sigma_{\text {eff }}$ that accounts for ion-induced mixing and sources of uncor- related convolution, such as sample roughness, and an exponential for the observed tailing, which has a characteristic decay length $\lambda_{\mathrm{d}}$. The Gaussian convolution function is

$$
G(z)=\frac{1}{(2 \pi)^{1 / 2} \sigma_{e f f}} \exp \left[-\frac{1}{2}\left(\frac{z}{\sigma_{e f f}}\right)^{2}\right] ; z \rightarrow(-\infty, \infty),
$$

and the exponential decay is described by

$$
F(\mathrm{z})=\frac{1}{\lambda_{\mathrm{d}}} \exp \left(-\frac{z}{\lambda_{\mathrm{d}}}\right) ; z \rightarrow(0, \infty) .
$$

$G(z)$ and $F(z)$ are numerically convoluted, using the Fourier transform method [20], with a Heaviside step function $\theta(z)$, where

$$
\theta(\mathrm{z})=0\left(-\infty, z_{1}\right) \text { and } \theta(\mathrm{z})=1 ;\left(z_{1}, \infty\right),
$$

to approximate the experimental profiles. These convoluted profiles are fit to the data with $\sigma_{\text {eff }}$ and $\lambda_{\mathrm{d}}$ as regression parameters. Results for $6.0 \mathrm{keV} \mathrm{Cs}{ }^{+}$and 5.5 $\mathrm{keV} \mathrm{O}_{2}^{+}$primary ion bombardment are summarized in Table 2. The best fit lineshapes are also plotted as solid lines in the graphs shown in Figure $2 \mathrm{c}$ and $\mathrm{d}$, clearly indicating that the combination of a Gaussian and exponential decay function yields excellent results. The effective location of the interface $z_{\mathrm{I}}$ was determined by the fit.

Finally, the interface between PS and PMMA is probed using the ${ }^{12} \mathrm{C}-\mathrm{PS}:{ }^{13} \mathrm{C}-\mathrm{PS} /{ }^{12} \mathrm{C}-\mathrm{PMMA}$ system with analysis using $6.0 \mathrm{keV} \mathrm{Cs}^{+}$primary ion bombardment. The nonmonotonic changes in the secondary ion yields for ${ }^{12} \mathrm{C},{ }^{12} \mathrm{C}^{1} \mathrm{H}$, and ${ }^{13} \mathrm{C}$ at the PS/PMMA interface shown in Figure 3, which have been observed previously [4], are not due to sample charging, as determined from analysis of ${ }^{12} \mathrm{C}$ secondary ion energy spectra at several depths through the sample. After $24 \mathrm{~h}$ of annealing at $125^{\circ} \mathrm{C}$, there is no apparent segregation of ${ }^{13} \mathrm{C}$-PS to the heterogeneous interface or free surface, as shown in Figure 3. Even though this is a lower temperature and annealing time than used with the hPS:dPS/hPMMA system (see Figure 1), it has been shown that a diffusion gradient and interfacial excess of $140 \mathrm{kDa}$ dPS (5\% vol/vol in $138 \mathrm{kDa}$ hPS) were readily visible for an hPS:dPS/hPMMA system after $4 \mathrm{~h}$ at $128^{\circ} \mathrm{C}$ [14]. The molecular weight dependency of diffusivity of entangled polymer chains in the melt (D $\alpha$ $\mathrm{M}_{\mathrm{w}}^{-2}$ ) is well known [21]. From this we can infer that discernible changes in the ${ }^{13} \mathrm{C}$-PS profile would be

Table 2. Convolution results

\begin{tabular}{cccccc}
\hline & $\begin{array}{c}(2 \sigma)_{84 / 16} \\
(\mathrm{~nm})\end{array}$ & $\begin{array}{c}\sigma_{50 / 16} \\
(\mathrm{~nm})\end{array}$ & $\begin{array}{c}\sigma_{84 / 50} \\
(\mathrm{~nm})\end{array}$ & $\begin{array}{c}\sigma_{\text {eff }} \\
(\mathrm{nm})\end{array}$ & $\begin{array}{r}\lambda_{\mathrm{d}} \\
(\mathrm{nm})\end{array}$ \\
\hline \hline $\mathrm{O}_{2}{ }^{+}$ & 19 & 7 & 12 & 4.2 & 10 \\
$\mathrm{Cs}^{+}$ & 14 & 5 & 9 & 1.7 & 9 \\
\hline
\end{tabular}




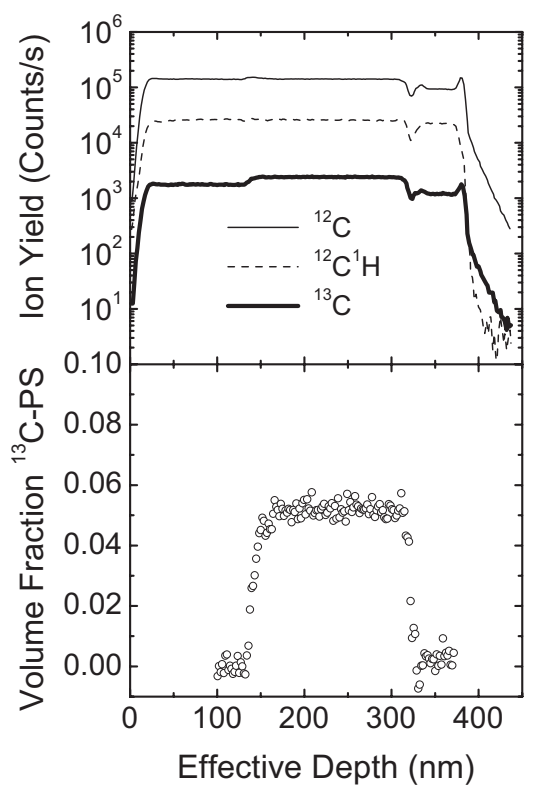

Figure 3. SIMS depth profile showing relative count rates and ${ }^{13} \mathrm{C}$-PS volume fraction of an analogous system to that shown in Figure 2, except that ${ }^{13} \mathrm{C}$-PS was used as the tracer instead of dPS. After $24 \mathrm{~h}$ at $125^{\circ} \mathrm{C}$, there is clearly no segregation of ${ }^{13} \mathrm{C}$-PS to the ${ }^{12} \mathrm{C}$-PS: $:{ }^{13} \mathrm{C}$-PS $/{ }^{12} \mathrm{C}$-PMMA interface, thereby proving that ${ }^{13} \mathrm{C}$ labeling does indeed provide a true tracer for depth profiling of polymer films and multilayers. SIMS analysis was performed using $6.0 \mathrm{keV} \mathrm{Cs}{ }^{+}$ion bombardment with detection of negative secondary ions. The effective depth was determined by assuming a constant PS sputtering rate throughout the analyzed film assembly.

observable at the ${ }^{12} \mathrm{C}-\mathrm{PS}:{ }^{13} \mathrm{C}$-PS $/{ }^{12} \mathrm{C}$-PMMA interface after $\sim 1 \mathrm{~h}$ at $125^{\circ} \mathrm{C}$, if there were an energetic preference for ${ }^{13} \mathrm{C}$-PS at the heterogeneous polymer/polymer interface. This is in stark contrast to the behavior witnessed at the hPS:dPS/hPMMA interface (see Figure 1 ), and is direct evidence that the use of ${ }^{13} \mathrm{C}$ labeling provides improved analysis conditions for tracer depth profiling using SIMS.

\section{Conclusions}

Significant changes in thermodynamic behavior due to deuterium labeling at an hPS:dPS/hPMMA interface have been shown [14], with the observation of segregation of dPS to the heterogeneous interface (see Figure 1). The molecular weight and annealing temperature of the hPS:dPS blend place this system well within the single phase region of the hPS:dPS phase diagram [10], which clearly demonstrates that the use of deuterium labeling may not provide a true tracer component for analysis using SIMS. This type of behavior could result in improper interpretation of experimental results due to labeling-induced artifacts. However, the use of ${ }^{13} \mathrm{C}$ labeling has been shown to be an exceptional alternative to deuterium labeling by providing a true tracer, with no observed segregation of ${ }^{13} \mathrm{C}-\mathrm{PS}$ to a ${ }^{12} \mathrm{C}-\mathrm{PS}:{ }^{13} \mathrm{C}-$ $\mathrm{PS} /{ }^{12} \mathrm{C}$-PMMA interface (see Figure 3 ). Although we don't anticipate the use of ${ }^{13} \mathrm{C}$ labeling to completely replace the use of deuterium labeling, it is expected that its use will indeed provide greatly improved analysis of various organic and biological systems.

\section{Acknowledgments}

This work was supported by the U.S. Department of Energy (DE-FG02-98ER45737). The authors gratefully acknowledge discussions with Professor Bruce Novak regarding the polymer synthesis and Dr. Dieter Griffis regarding the SIMS analysis. The TOF SIMS (PHI TRIFT I) measurements were performed by Dr. Zhengmao Zhu at the Analytical Instrumentation Facility, North Carolina State University (Raleigh, NC).

\section{References}

1. Winograd, N. The Magic of Cluster SIMS. Anal. Chem. 2005, 77, 142A-149A

2. Weibel, D.; Wong, S.; Lockyer, N.; Blenkinsopp, P.; Hill, R.; Vickerman, J. C. A C60 Primary Ion Beam System for Time of Flight Secondary Ion Mass Spectrometry: Its Development and Secondary Ion Yield Characteristics. Anal. Chem. 2003, 75, 1754-1764.

3. Szakal, C.; Sun, S.; Wucher, A.; Winograd, N. C60 Molecular Depth Profiling of a Model Polymer. Appl. Surf. Sci. 2004, 231, 183-185.

4. Harton, S. E.; Stevie, F. A.; Ade, H. Secondary Ion Mass Spectrometry Depth Profiling of Amorphous Polymer Multilayers Using $\mathrm{O}_{2}^{+}$and $\mathrm{Cs}^{+}$ Ion Bombardment with a Magnetic Sector Instrument. J. Vac. Sci. Technol. A 2006, 24, 362-368.

5. Gillen, G.; Hues, S. M. Doped Gelatin Films as a Model Matrix for Molecular Secondary Ion Mass-Spectrometry Studies of Biological SoftTissue. J. Am. Soc. Mass Spectrom. 1993, 4, 419-423.

6. Jones, R. A. L. Polymers at Surfaces and Interfaces; Cambridge University Press: New York, 1999; p. 56.

7. Schwarz, S. A.; Wilkens, B. J.; Pudensi, M. A. A.; Rafailovich, M. H.; Sokolov, J.; Zhao, X.; Zhao, W.; Zheng, X.; Russell, T. P.; Jones, R. A. L. Studies of Surface and Interface Segregation in Polymer Blends by Secondary Ion Mass Spectrometry. Mol. Phys. 1992, 76, 937-950.

8. Atkin, E. L.; Kleintjens, L. A.; Koningsveld, R.; Fetters, L. J. LiquidLiquid Phase Separation in Multicomponent Polymer Systems. 21. Deuterium-Isotope Effect in Polymer Compatibility. Macromol. Chem. 1984, 185, 377-387.

9. Russell, T. P. Changes in Polystyrene and Poly(methyl methacrylate) Interactions with Isotopic Substitution. Macromolecules 1993, 26, 5819.

10. Bates, F. S.; Wignall, G. D. Isotope-Induced Quantum-Phase Transitions in the Liquid State. Phys. Rev. Lett. 1986, 57, 1429-1432.

11. Jones, R. A. L.; Kramer, E. J.; Rafailovich, M. H.; Sokolov, J.; Schwarz, S. A. Surface Enrichment in an Isotopic Polymer Blend. Phys. Rev. Lett. $1989,62,280-283$.

12. Hariharan, A.; Kumar, S. K.; Rafailovich, M. H.; Sokolov, J.; Zheng, X.; Duong, D. H.; Schwarz, S. A.; Russell, T. P. The Effect of Finite Film Thickness on the Surface Segregation in Symmetrical Binary Polymer Mixtures. J. Chem. Phys. 1993, 99, 656-663.

13. Strazielle, C.; Benoit, H. Some Thermodynamic Properties of PolymerSolvent Systems Comparison between Deuterated and Undeuterated Systems. Macromolecules 1975, 8, 203-205.

14. Harton, S. E.; Stevie, F. A.; Ade, H. Investigation of the Effects of Isotopic Labeling at a PS/PMMA Interface using SIMS and Mean-Field Theory. Macromolecules 2006, 39, 1639-1645.

15. Mahoney, C. M.; Roberson, S. V.; Gillen, G. Depth Profiling of 4-Acetamindophenol-Doped Poly(lactic acid) Films Using Cluster Secondary Ion Mass Spectrometry. Anal. Chem. 2004, 76, 3199-3207.

16. Xia, J.; Matyjaszewski, K. Controlled/“Living" Radical Polymerization. Atom Transfer Radical Polymerization Using Multidentate Amine Ligands. Macromolecules 1997, 30, 7697-7700.

17. Wilson, R. G.; Stevie, F. A.; Magee, C. W. Secondary Ion Mass Spectrometry: A Practical Handbook for Depth Profiling and Bulk Impurity Analysis; John Wiley and Sons: New York, 1989; pp. 2.11-1-2.11-4.

18. Dowsett, M. G.; Barlow, R. D.; Allen, P. N. Secondary-Ion MassSpectrometry Analysis of Ultrathin Impurity Layers in Semiconductors and Their Use in Quantification, Instrumental Assessment, and Fundamental Measurements. J. Vac. Sci. Technol. B 1994, 12, 186-198.

19. Allen, P. N.; Dowsett, M. G. Maximum-Entropy Quantification of Sims Depth Profiles Behavior as a Function of Primary Ion Energy. Surf. Interface Anal. 1994, 21, 206-209.

20. Press, W. H.; Flannery, B. P.; Teukolsky, S. A.; Vetterling, W. T. Numerical Recipes in Fortran 77: The Art of Scientific Computing; Cambridge University Press: New York, NY 1992; pp. 498-499.

21. de Gennes, P. G. Reptation of a Polymer Chain in Presence of Fixed Obstacles. J. Chem. Phys. 1971, 55, 572-579. 